\title{
Grinding Metre-Scale Mirror Segments for the E-ELT Ground Based Telescope
}

\author{
Authors: P. Comley, P. Morantz, P. Shore (2), X. Tonnellier \\ Cranfield University Precision Engineering Centre, Cranfield University, MK43 0AL, UK
}

\begin{abstract}
:
The next generation of ground based telescopes require many hundreds of metre scale off-axis mirrors. In this paper the grinding of a 1.45 metre scale Zerodur ${ }^{\circledR}$ mirror segment for the European Extremely Large Telescope (E-ELT) is introduced. Employing an R-theta grinding mode with a multi stage grinding process material removal rates of up to $187.5 \mathrm{~mm}^{3} / \mathrm{s}$ are achieved, whilst typically removing up to $1 \mathrm{~mm}$ depth of material in total. Results show a RMS form error of $<1 \mu \mathrm{m}$, with subsurface damage $<10 \mu \mathrm{m}$, and a production cycle time of under 20 hours.
\end{abstract}

Keywords: Grinding, Optical, Surface Integrity

\section{Introduction}

Modern science programmes require increasingly large telescopes. Monolithic mirrors are limited to around $10 \mathrm{~m}$ for reasons of manufacturing, measurement, and transportation. Consequently segmented mirror technology, as applied to the Keck telescopes has become the main basis for scaling up. EELT - figure 1 - and TMT are next generation ground based telescopes presently under development which will require many hundreds of metre scale off-axis mirrors [1, 2, 3]. These segments will be made from low expansion glass or glass ceramics such as $\mathrm{ULE}^{\circledR}$, Zerodur $^{\circledR}$, Astrositall ${ }^{\circledR}$ or Clearceram ${ }^{\circledR}$. Their successful fabrication places extreme demands on the manufacturing process chain operations, amongst which is grinding, demanding high form accuracy, low sub surface damage and roughness, and critically faster processing times. At $42 \mathrm{~m}$ in diameter the E-ELT primary mirror is constructed from 984 hexagonal segments, each $1.45 \mathrm{~m}$ wide. With a target completion date of 2018 even conservative estimates place the required production rate for segments at 1 per day.

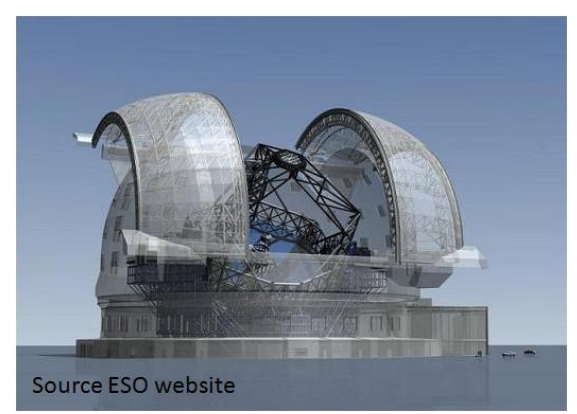

Figure 1 - Illustration of proposed E-ELT telescope

Present 'state of the art' manufacturing technologies for providing metre scale ultra precision mirrors are associated with processing times of hundreds of hours, consequently the time to manufacture hundreds or even thousands of mirrors for such projects as E-ELT would involve many years of production.

In order to reduce manufacturing time for large mirrors, a new UK based research initiative was established with the prime objective to reduce processing time of large scale complex shape surfaces [4]. The research proposed a manufacturing chain with rapid low-damage precision grinding followed by an iterative polishing stage and finally reactive atom plasma figuring, see figure 2 .

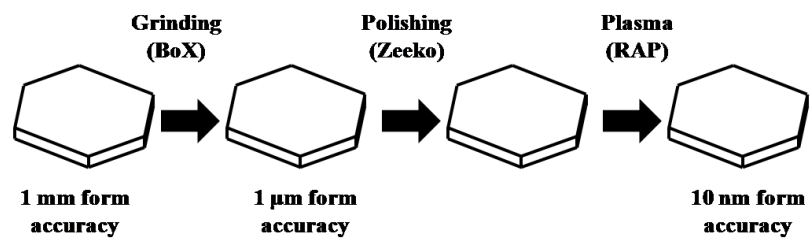

Figure 2 - Proposed process chain for manufacturing large optics

During the late 1970's high precision diamond turning machines were devised to produce large optics in the 1-2 metre range $[5,6]$. These were based on proven precision engineering principles [7] to achieve high motion repeatability with minimal errors. Further enhancements included the use of metrology frames, providing motion accuracies in the order of 1 part in $10^{6}$. In the 1980's machines such as the OAGM - off axis grinding machine - built for Eastman Kodak [8] had a 2.5 m capacity with a grinding accuracy of approaching 1 part in $10^{6}$. Such state of the art machines today require high levels of investment for both acquisition and operation, and do not offer the levels of throughput envisaged for the next generation of ground based telescopes. Parallel research during this period led to the development of novel machine tool structures, leading to highly stiff and well damped grinding machines such as the Tetraform [9]. These machines demonstrated high performance grinding on small scale parts. The Cranfield BoX grinding machine has been developed based on this philosophy [10] to provide metre scale grinding capability for optics at high removal rates whilst minimising levels of sub-surface damage (SSD). This ideally places BoX to meet the demands of the proposed process chain; figure 3 illustrates how the chain relates to other manufacturing technologies. 


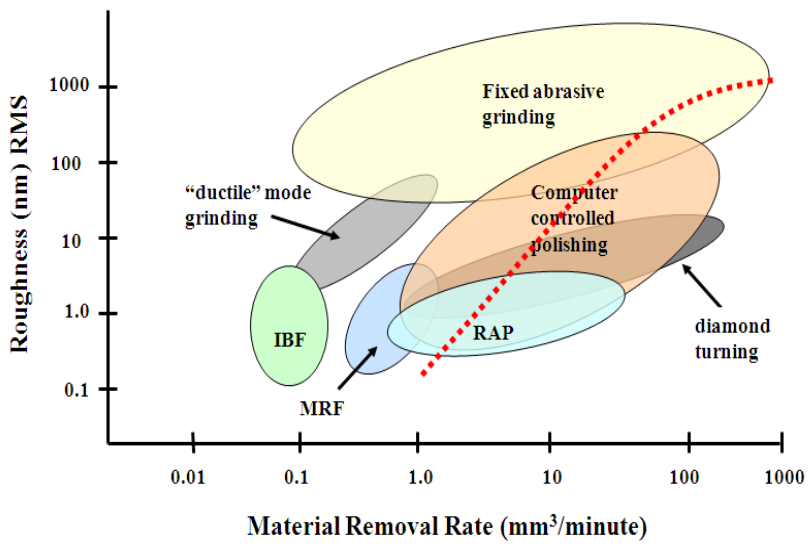

Figure 3 - Manufacturing Chains

In this paper the grinding of a 1.45 metre scale mirror segment for the European Extremely Large Telescope (E-ELT) is introduced. The segment is one of 7 prototypes for the M1 (primary) mirror being manufactured under contract for ESO. Grinding has been carried out within the Loxham large optics aspherisation facility at Cranfield, part of the Precision Engineering Centre. The facility includes a BoX machine for grinding, Leitz pmmf for large scale metrology, reactive atom plasma (RAP) machine for final form figuring, together with the specialist handling and fixturing systems essential for the safe and precise handling of large glass segments.

\section{High Performance Precision Grinding}

To grind ceramics and glasses rapidly with good form accuracy, high levels of 'loop' stiffness are required between the grinding wheel and workpiece. Static loop stiffness is key to the transfer of precise machine motions to the component surface profile. This is especially important at the workpiece edges where low stiffness will result in edge 'roll off' as the contact area between the grinding wheel and workpiece reduces. Dynamic loop stiffness is central to the control of abrasive penetration required to reduce excessive crack propagation. Higher levels of dynamic loop stiffness will counter the higher force and force variation associated with increased material removal rates. The control of abrasive penetration depth should be achieved at frequencies well above the operational frequency of the grinding spindle in order to prevent irregular levels of SSD, caused through excitation by the spindle rotation [11].

In order to achieve sub-micron levels of form accuracy over metre scale surfaces it is necessary to ensure machine motions are highly repeatable and are configured for error correction and compensation techniques.

\section{BoX Grinding Machine}

The Cranfield BoX ultra precision grinding machine, see figure 4 , has been designed for manufacturing high precision low slope surfaces. The machine has a novel configuration and construction with high static and dynamic loop stiffness, together with a compact footprint. To minimise the moving masses, machine motions are achieved using three fundamental axes. Two stacked hydrostatic linear axes $(\mathrm{x}, \mathrm{z})$ holding the inclined hydrostatic grinding spindle and a single hydrostatic rotary spindle (c) supporting the workpiece.

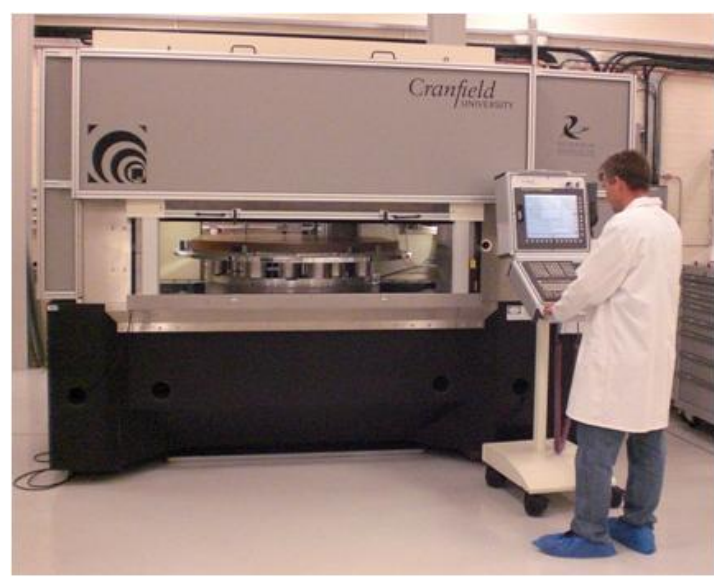

Figure 4 - BoX machine

To achieve good thermal stability, stray heat transfer from the grinding spindle and linear motors was minimised using high diffusivity materials and active temperature control of motors. Zerodur gratings were utilised to reduce thermal creep. The hydrostatic oil was maintained within $+/-0.1{ }^{\circ} \mathrm{C}$ using active feedback and feed forward temperature control. Temperature control for linear and rotary motors and grinding coolant was also provided. A non-stressed metrology frame is incorporated into the design using a LVDT probe referenced to a straight edge using a laser interferometer. This system provides the capability to carry out in-situ measurement against a traceable artefact for error compensation application.

The machine configuration employs an R-theta grinding mode with an inclined toroidal shape diamond grit cup wheel. The $325 \mathrm{~mm}$ external diameter of the wheel is selected to provide a grinding speed in the range $25-35 \mathrm{~m} / \mathrm{s}$ within a rotational frequency range of $25-35 \mathrm{~Hz}$. The radius of curvature of the wheel is determined by the geometric contact area between wheel and workpiece. For free-form surfaces as the local slope of the surface changes across and around the workpiece, then the contact zone on the grinding wheel moves around the periphery. The grinding mode therefore demands a control system and algorithm strategy to accommodate this changing cutting region and peripheral contact zone [12].

\section{Metrology}

Off machine metrology for the free-form surfaces was undertaken on a Leitz pmmf. With a capacity of $3 \mathrm{~m} \times 2 \mathrm{~m} \times 1 \mathrm{~m}$ and equipped with a low force sensing head the machine has a traceable accuracy of $1.9+(\mathrm{L} / 400)$ microns. Tests indicated that micron level repeatability is achievable on this instrument on low slope artefacts using scanning routines, within a $+/-1 \quad{ }^{\circ} \mathrm{C}$ controlled environment. High density scan data was postprocessed to produce topographical mapping of the ground surfaces.

\section{Segment Grinding}

Grinding performance has been demonstrated on a number of materials including Zerodur, ULE, fused silica and silicon carbide. Zerodur and ULE are amongst the materials selected for use in the prototype development phase of the E-ELT programme. Using a multi-stage grinding process, high material removal rates, up to $187.5 \mathrm{~mm}^{3} / \mathrm{s}$, with form accuracies of $+/-1$ $\mu \mathrm{m}$ peak to valley were achieved on spherical Zerodur and ULE 
parts of up to $1 \mathrm{~m}$ diameter. Surface roughness was 100-200 nm RMS and SSD below $10 \mu \mathrm{m}[13]$.

The multistage grinding process includes rough, semi-finish and finish stages, using D76, D46 and D25 diamond grit size respectively. The resin bond wheels are formed and dressed insitu using machine integrated systems. Forming is carried out using preformed electroplated diamond grit wheels, and dressing with an Aluminium oxide compound.

To validate the free-form grinding capability prior to grinding the E-ELT segment, an off-axes spherical part was ground, then subsequently polished to demonstrate the ground surface suitability for post-processing. The part a $350 \mathrm{~mm}$ diameter, $60 \mathrm{~mm}$ thick, $2.9 \mathrm{~m}$ radius of curvature component, mounted $19.2 \mathrm{~mm}$ off-centre was devised to represent a programming and machine performance challenge, far exceeding than that posed by specification of the E-ELT segment. The surface form was measured on the CMM, figure 5 shows a surface map of the ground surface.

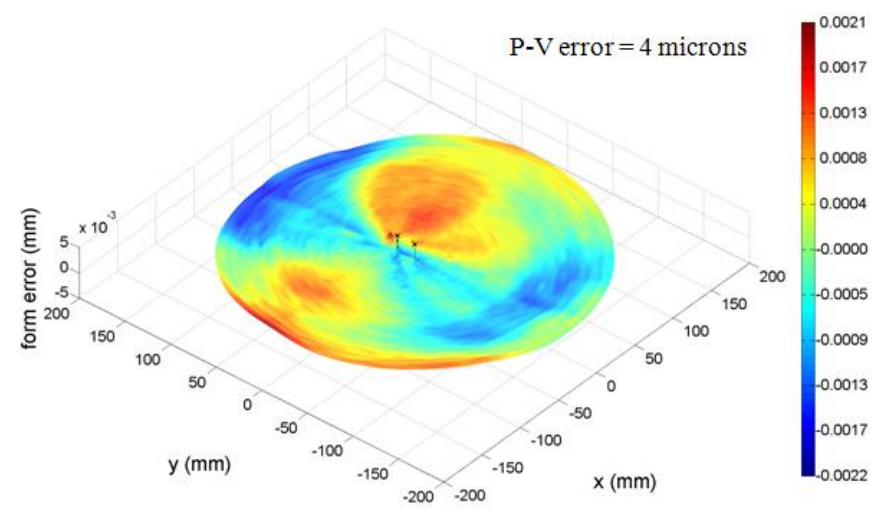

Figure 5 - Form departure for 2.9 m ROC sphere ground 19.2 off-centre

The total form accuracy was $4 \mu \mathrm{m}$ peak-valley for this highly free-form part. The mid-spatial errors over a $20-50 \mathrm{~mm}$ wavelength were below $300 \mathrm{~nm}\left(\mathrm{P}_{\mathrm{t}}\right)$ in the radial orientation and substantially less circumferentially. Subsequently smoothing and polishing operations demonstrated the suitability of the ground surface quality for final figure correction.

The hexagonal E-ELT segment selected for grinding was one of the $984 \mathrm{M} 1$ mirror segments. Each segment is to $1.45 \mathrm{~m}$ wide and $50 \mathrm{~mm}$ thick with a central cavity $200 \mathrm{~mm}$ diameter and $36 \mathrm{~mm}$ depth on the underside. The selected segment lies on the periphery of the M1 (mirror), see figure 6, which has an ellipsoidal form as described by equation 1 . The segment sag is $3 \mathrm{~mm}$ and departure from spherical $\sim 150 \mu \mathrm{m}$, with form symmetry about the $\mathrm{X}$-axis.

$$
z(x, y)=\frac{\left(\frac{1}{R}\right)\left(x^{2}+y^{2}\right)}{\left[1+\sqrt{1-(1+k)\left(\frac{1}{R}\right)^{2}\left(x^{2}+y^{2}\right)}\right]}
$$

Equation 1

Where:

$\mathrm{R}=84 \mathrm{~m}$

$\mathrm{k}=-0.993295$

(Base radius of curvature)

(Conic constant)

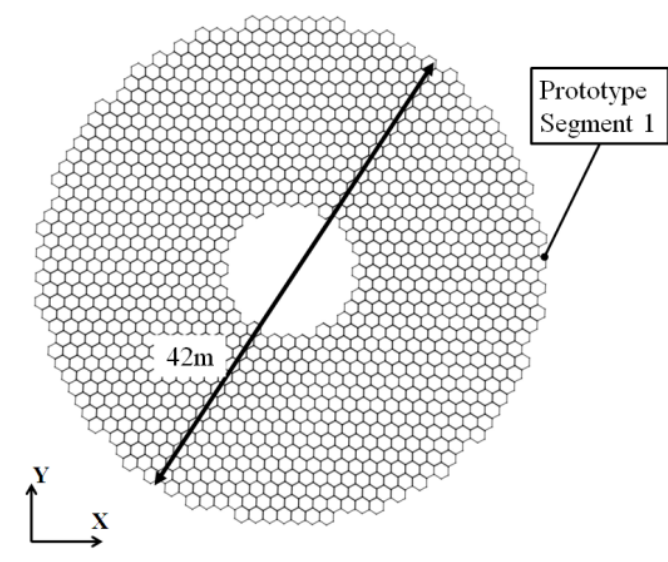

Figure 6 - E-ELT Primer mirror (M1) segment array

The required form accuracy from the grinding operation was $8 \mu \mathrm{m}$ peak-valley, with a minimal mid spatial signature. Specified reference features were the rear central cavity $(x, y)$, underside ( $\mathrm{z}$ ) and one of the hexagonal edges (rotation).

The part was procured rough ground with an $84 \mathrm{~m}$ radius spherical surface leaving $1 \mathrm{~mm}$ of material to be removed. The part was characterised on the CMM using the specified reference features to establish a co-ordinate system. Measurements were then referenced to master features on the grinding support system, see figure 8 . The initial stage of the BoX grinding corrected gross form error whilst removing the SSD present from the preceding aggressive rough grinding regime, typically 200$300 \mu \mathrm{m}$. Finish grind parameters were applied and a CMM measurement undertaken to confirm geometry and compensation algorithms. The final grinding passes were subsequently made with a D25 wheel with the error compensation algorithms applied. Figure 7 shows the segment undergoing grinding.

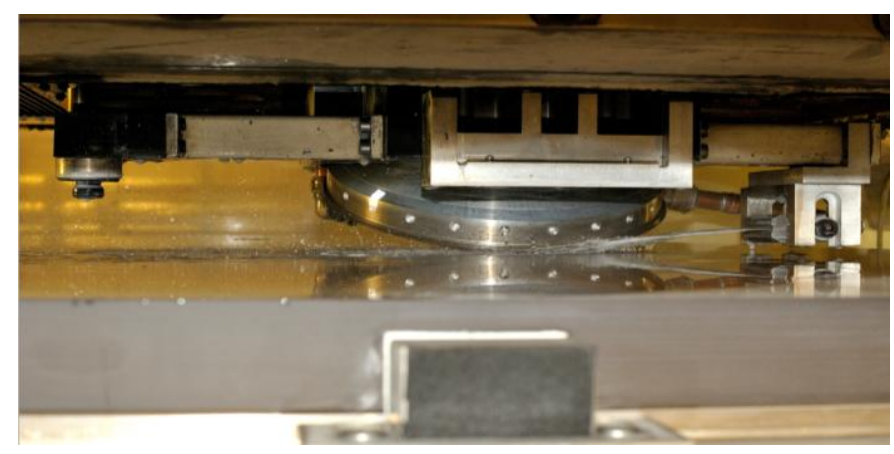

Figure 7 - E-ELT Segment undergoing grinding

Figure 8 shows the surface being measured on the CMM. The same reference features were used to re-establish the coordinate system and the scan data was processed to create a surface map showing deviation against the surface form as described by equation 1 . In excess of 500,000 measurement points were taken to within $0.5 \mathrm{~mm}$ of the edges. 


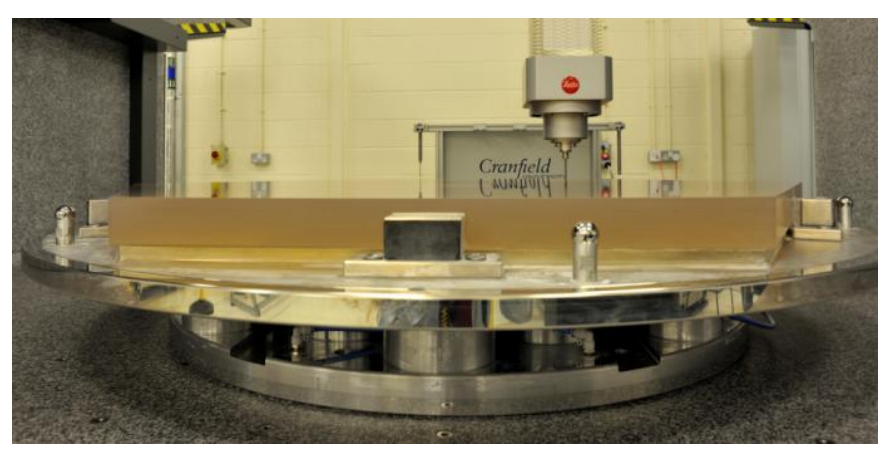

Figure 8 - E-ELT Segment metrology

The surface map of the ground segment is shown in figure 9. The results show a surface form error of RMS $<1 \mu \mathrm{m}$, with a P-V $<$ $5.5 \mu \mathrm{m}$, with no detectable discontinuity resulting from the crosssectional variation over the central cavity region. The segment thickness was held to within $3 \mu \mathrm{m}$ of nominal specification (target $100 \mu \mathrm{m}$ ) with no visible edge roll-off or edge chipping as a result of grinding.

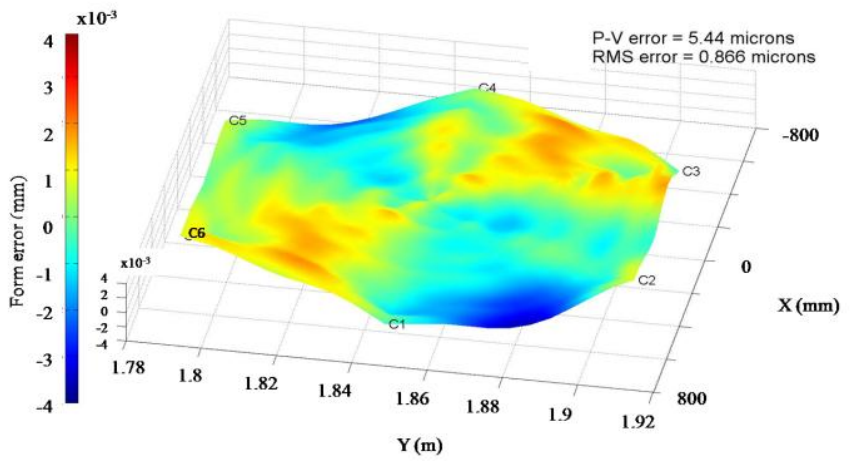

Figure 9 - Surface map of ground E-ELT segment

\section{Discussion}

Building on research previously carried out on $350 \mathrm{~mm}$ and 1 metre parts, the grinding technology has been applied for $1.5 \mathrm{~m}$ components. The high 'loop' stiffness of the BoX machine is demonstrated by the absence of edge roll-off and chipping (static loop stiffness) and sub $10 \mu \mathrm{m}$ levels of SSD (dynamic loop stiffness). The multistage grinding process enables material removal rates of up to $187.5 \mathrm{~mm}^{3} / \mathrm{s}$ to be achieved, resulting in production cycle times of under 20 hours to process E-ELT 1.45 $\mathrm{m}$ segments, removing up to $1 \mathrm{~mm}$ of material. Results show a RMS form error of $<1 \mu \mathrm{m}$ for an error compensated grind with surface roughness of between $100-200 \mathrm{~nm} \mathrm{R}_{\mathrm{a}}$

The grinding process offers lower cycle times than that currently being reported together with high accuracy and low levels of SSD. With improved input quality, cycle times for subsequent process stages such as computer controlled polishing will be dramatically reduced. The capability therefore offers a competitive cost advantage for the large scale production required for telescope, fusion power and other large scale optics.

\section{Conclusion}

Grinding technology has been demonstrated for $1.45 \mathrm{~m}$ freeform Zerodur segments, with far higher fabrication rates than that of the currently reported state of the art. Surfaces produced have extremely low levels of subsurface damage $<10 \mu \mathrm{m}$. The reported capability is ideally placed to meet the future large scale production requirements for ground based telescope such as EELT.

\section{Acknowledgements}

The authors gratefully acknowledge project funding support through the UK Joint Research Councils - Basic Technologies programme and EPSRC funded Integrated Knowledge Centre for Ultra Precision Structured Surfaces (UPS ${ }^{2}$.

\section{References}

[1]. L. M. Stepp, S.E. Strom, 2004, The Thirty Metre Telescope project design and development phase, Proc SPIE, Vol. 5382, Pg 67-75

[2]. R. Gilmozzi, 2004, Science and technology drivers for future giant telescopes, Proc SPIE, Vol. 5489, Pg 1-10

[3]. P.Shore, et al, 2010, Precision engineering of astronomy and gravity research (keynote), Journal of Manufacturing Technology, CIRP Annals, 59/2, pg 694-716.

[4]. Shore, P., May-Miller, R., 2003, Production Challenge of the Optical Segments for Extra Large Telescopes, Proc International Workshop on extreme optics and sensors 40,25.

[5]. Donaldson, R. R., Patterson, S.R., 1983, Design and construction of a vertical axis diamond turning machine (LODTM), Proc SPIE, UCRL 89738, Vol 433, Pg62-67

[6]. Wills-Moren, W.J., et al., 1982, Some aspects of the design and development of a high precision diamond turning machine, Annals of CIRP, vol31/1, pg 409.

[7]. McKeown, P.A., Loxham, J., 1973, Some aspects of the design of high precision measuring machines, Annals of CIRP, Vol 22/1, pg 139.

[8]. Wills-Moren, W.J., Wilson, T. 1989, The design and manufacture of a large CNC grinding machine for off-axis mirror segments, Annals of CIRP, Vol 38/1, pg529.

[9]. Stephenson, D.J., Corbett, J., Hedge, J., 2003, Precision grinding using Tetraform C, STIM Manufacturing Engineering No.5, pg30-34, ISSN 0869-7566.

[10]. P.Shore, P.Morantz, X.Luo, X.Tonnellier, R.Read, R.May-Miller, 2005, Design Philosophy of the ultra precision big OptiX BoX machine, Laser Metrology and Machine Performance VII, pg200-209, ISBN: 1861941-18-8

[11]. Tonnellier, X., 2009, Precision machining of large freeform optics, $\mathrm{PhD}$ Thesis, Cranfield University.

[12]. Shore, P. et al., 2005, Grinding mode of the "BoX " ultra precision freeform grinder, ASPE Proceedings of the Annual Meeting, Oct9-14, Pg114-117, ISBN 1-887706-39-9

[13]. Tonnellier, T. et al. 2008, Sub-surface damage issues for effective fabrication of large optics, Proc of SPIE Vol. 7018, pg 701836-1 to 701836-10, ISBN 9780819472281 